\title{
On the oscillons in the signum-Gordon model
}

Z. Świerczyński

To cite this article: Z. Świerczyński (2017) On the oscillons in the signum-Gordon model, Journal of Nonlinear Mathematical Physics 24:1, 20-28, DOI:

https://doi.org/10.1080/14029251.2016.1274112

To link to this article: https://doi.org/10.1080/14029251.2016.1274112

Published online: 04 January 2021 


\title{
On the oscillons in the signum-Gordon model
}

\author{
Z. Świerczyński \\ Institute of Computer Science, Pedagogical University, Podchorażych 2 \\ Cracow, 30-084, Poland \\ zs@up.krakow.pl
}

Received 23 July 2016

Accepted 3 November 2016

\begin{abstract}
New periodic solutions of signum-Gordon equation are presented. We first find solutions $\varphi_{0}(x, t)$ defined for $(x, t) \in \mathbb{R} \times[0, T]$ and satisfying the condition $\varphi_{0}(x, 0)=\varphi_{0}(x, T)=0$. Then these solutions are extended to the whole spacetime by using (2.4).
\end{abstract}

Keywords: Nonlinear wave equation; periodic solution.

2000 Mathematics Subject Classification: 35L05, 35L70

\section{Introduction}

The signum-Gordon model is probably the simplest system with a V-shaped potential. The potential in these models is not smooth in its minimum (in the signum-Gordon model the potential is the absolute value of the field). Some issues concerning these models as for example self-similar solutions, Q-balls, or static point charges were investigated, but understanding of these models is poorer than ones with smooth potentials [1,2]. One of the features of these models is that localised field configurations have no exponential tails characteristic for models with massive fields. The fields forming a Q-ball in complex signum-Gordon model or surrounding a static point charge in signum-Gordon model vanish outside some bounded region.

In [3] an oscillating solution in 1+1 dimensional signum-Gordon equation was discovered. Oscillating field configurations called oscillons are not rare in models with smooth potentials. They are localised in space and long lived, but lose their energy through the radiation and after some time collapse $[5,8,9,12-15]$. The situation is different if one is interested in localised, strictly timeperiodic solutions $[6,7,10,11,17]$. The only such a solution in $1+1$ dimensional Klein-Gordon model with real scalar field and analytic potential is the breather in the sine-Gordon equation [16]. In the models in spacetime containing no more than three spatial dimensions one can find strictly time-periodic weakly localised solutions possessing a core whose energy density is much larger than the energy density of the tail. The tail has standing wave asymptotic and contains infinite energy. The solutions with the minimal amplitude of the standing wave tail are called quasi-breathers $[8,9]$. The cores of quasi-breathers are very similar to the cores of oscillons.

The mentioned above oscillating solution in signum-Gordon model does not radiate and it is strictly periodic, so it should be named breather. Nevertheless, the authors of [3] called it oscillon and we will also use this name. The solution has no exponential tail. The field differs from its vacuum value only on some finite interval. The length of this interval we will call the size of the 


\section{Z. Swierczyński / On the oscillons in the signum-Gordon model}

oscillon. Since signum-Gordon equation possesses a scale invariance there is a one-parameter family of oscillons parametrized by the oscillon size. We omit in our considerations solutions describing oscillons moving with constant velocity, which may be obtained trivially by applying Lorentz transformations (boosts). The period of the oscillon is equal to its size and the energy is proportional to third power of the size.

In [4] more periodic solutions were found. The boundaries of these oscillons (the endpoints of the interval containing the oscillon) perform periodic motion. The boundaries move with a constant velocity during the time equal to the half the oscillon period. Then the direction of their motion changes to opposite one. These solutions exist for all values of the velocity of the motion of the boundaries not exceeding the speed of light. If the velocity is equal to zero (the boundaries do not move), the solution found in [3] is obtained. The energy of the oscillon depends only on its size; two oscillons whose boundaries move with different velocities but have the same size have also the same energy.

In the next section we present new periodic solutions of the signum-Gordon equation. The solutions found in [4] may be considered as a special cases of these new ones.

\section{Periodic solutions}

The field in $1+1$ dimensional signum-Gordon model satisfies the equation

$$
\frac{\partial^{2} \varphi}{\partial t^{2}}-\frac{\partial^{2} \varphi}{\partial x^{2}}=-\lambda \operatorname{sign}(\varphi)
$$

where the sign function takes value -1 when its argument is negative, 0 when the argument is equal to 0 , and 1 for positive values of the argument. Investigating or constructing a solution of this equation, one may divide the space-time into regions in such a way that the sign of $\varphi$ is the same for all points in each region, so in any of these regions the right hand side of (2.1) is constant. Fitting together solutions found in different regions we require the field $\varphi(x, t)$ to have continuous derivatives. This implies that the energy density is also continuous. We shall consider weak solutions and do not assume that the second derivatives exist everywhere [4]. The field $\varphi(x, t)$ is assumed to be a $C^{1}$ function satisfying

$$
\int d t d x\left(\left(\frac{\partial^{2} \psi}{\partial t^{2}}-\frac{\partial^{2} \psi}{\partial x^{2}}\right) \varphi+\lambda \psi \operatorname{sign}(\varphi)\right)=0
$$

for arbitrary $C^{2}$ test function $\psi$ with a compact support. The singularities of the second derivatives occur not only on the boundaries of the regions containing parts our solution with a fixed sign of the field value, but also may propagate through these regions with the speed of light. Such a behaviour of the singularities may be connected with the corners of the region boundary.

It was observed in [4] that a solution $\varphi_{0}(x, t)$ of $(2.1)$ defined for $(x, t) \in \mathbb{R} \times[0, T]$ and satisfying

$$
\varphi_{0}(x, 0)=\varphi_{0}(x, T)=0
$$

may be continued to a periodic solution with the period $2 T$

$$
\varphi(x, t)=\left\{\begin{array}{rc}
\varphi_{0}(x, t-k T), & k T \leq t \leq(k+1) T \\
& k=\cdots-2,0,2,4, \ldots \\
-\varphi_{0}(x,(k+1) T-t), & k T \leq t \leq(k+1) T \\
k & =\cdots-1,1,3, \ldots
\end{array}\right.
$$


In order to find appropriate function $\varphi_{0}$ we shall consider the initial data

$$
\begin{gathered}
\varphi_{0}(x, 0)=0, \\
\frac{\partial \varphi_{0}}{\partial t}(x, 0)=\left\{\begin{array}{cl}
0 & \text { if } x<0, \\
\chi(x) & \text { if } 0 \leq x \leq 2 T, \\
0 & \text { if } x>2 T,
\end{array}\right.
\end{gathered}
$$

where $\chi$ is a continuous function with piecewise continuous derivative, $\chi(x)>0$ for $0<x<2 T$ and $\chi(0)=\chi(2 T)=0$. We assume that $\varphi_{0}$ is positive between two timelike curves $\gamma_{a}$ and $\gamma_{d}$ (see Fig. 1). The curve $\gamma_{a}$ connects the point $(0,0)$ with the point $(\Delta, T),|\Delta|<T$. The curve $\gamma_{d}$ connects point $(2 T, 0)$ with point $\left(2 T+\Delta_{2}, T\right)$. We will see later that $\Delta_{2}=\Delta$ and that the curve $\gamma_{d}$ may be obtained by a translation of $\gamma_{a}$ along the $x$ axis (see Fig. 1). This implies that the oscillon size remains constant in time. Outside the region contained between the curves $\gamma_{a}$ and $\gamma_{d}$ the field $\varphi_{0}(x, t)$ is equal to zero.

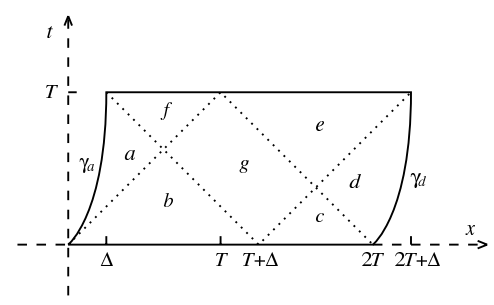

Fig. 1. Support of $\varphi_{0}$. The field $\varphi_{0}$ is different from zero (positive) in the region surrounded by the solid line.

In the region where the field $\varphi_{0}$ is positive, it satisfies the equation

$$
\frac{\partial^{2} \varphi}{\partial t^{2}}-\frac{\partial^{2} \varphi}{\partial x^{2}}=-\lambda
$$

Solution of (2.7) in the triangle $t<x<2 T-t, 0<t<T$ (parts " $b$ ", " $c$ ", and " $g$ " in Fig. 1 ) with the initial data (2.5), (2.6) is given by

$$
\varphi_{0}(x, t)=H(x+t)-H(x-t)-\frac{\lambda}{2} t^{2},
$$

where $d H(x) / d x=\chi(x) / 2$. Condition (2.3) implies

$$
H(2 T)=H(0)+\frac{\lambda}{2} T^{2}
$$

This solution can be extended to the set $A=\{(x, t):-t \leq x \leq t, 0 \leq t \leq T\}$ in such a way, that (2.3) is satisfied. The extension, which we denote by $\tilde{\varphi_{0}}$, may be expressed in terms of the function $H$

$$
\tilde{\varphi_{0}}(x, t)=H(x+t)-H(x-t+2 T)+\frac{\lambda}{2}\left(T^{2}-t^{2}\right),
$$

if $-t \leq x \leq t, 0 \leq t \leq T$ (the part " $P$ ” in Fig. 2),

$$
\tilde{\varphi_{0}}(x, t)=H(x+t-2 T)-H(x-t)+\frac{\lambda}{2}\left(T^{2}-t^{2}\right)
$$

if $2 T-t \leq x \leq 2 T+t, 0 \leq t \leq T$ (the part " $R$ ” in Fig. 2). 


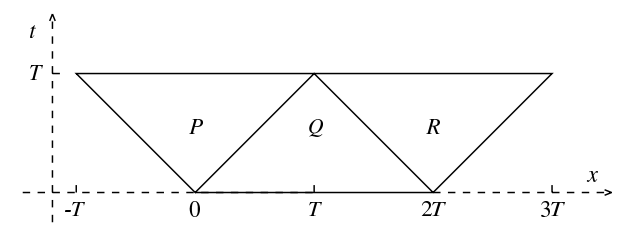

Fig. 2. Domain of $\tilde{\varphi_{0}}$.

If we introduce the function

$$
\tilde{H}(x)=\left\{\begin{array}{cc}
H(x+2 T)-\frac{\lambda}{2} T^{2} & \text { if }-2 T \leq x<0, \\
H(x) & \text { if } 0 \leq x \leq 2 T \\
H(x-2 T)+\frac{\lambda}{2} T^{2} & \text { if } 2 T<x \leq 4 T
\end{array}\right.
$$

then (2.8), (2.10), and (2.11) may be written as

$$
\tilde{\varphi_{0}}(x, t)=\tilde{H}(x+t)-\tilde{H}(x-t)-\frac{\lambda}{2} t^{2} .
$$

The function $\chi$ is continuous and has piecewise continuous derivative. It follows that $\tilde{H}$ is a $C^{1}$ function with piecewise continuous second derivative. From (2.13) we see that if the second derivative of the function $\tilde{H}$ does not exist at some point, then the second derivatives of the function $\tilde{\varphi_{0}}$ have propagating with the speed of light singularities. The conditions which we will impose later on the function $\chi$ (see (2.20), (2.21)) imply that the second derivative of the function $\tilde{H}$ does not exist at the points $\Delta-T, 0, T+\Delta, 2 T$. The corresponding singularities of the second derivatives of the $\tilde{\varphi_{0}}$ propagate along the lines $x=t, x=T+\Delta-t, x=T+\Delta+t, x=2 T-t$ (the dotted lines in Fig. 1).

Constructing the solution $\varphi_{0}$ we restrict the function $\tilde{\varphi_{0}}$ to the region contained between curves $\gamma_{a}$ and $\gamma_{d}$. Then we extend it using the vacuum solution $\varphi \equiv 0$ on the left- and right- hand sides of this region. Thus our solution $\varphi_{0}$ is given by (2.8) in parts " $b$ ", " $c$ ", " $g$ ", (2.10) in parts " $a$ ", " $f$ ", and (2.11) in parts " $d$ ", " $e$ ". Outside all these parts (on the rest of the strip $\mathbb{R} \times[0, T]) \varphi_{0}$ is equal to zero. The function $\tilde{\varphi_{0}}$ and the vacuum match only if the function $\tilde{\varphi_{0}}$ vanishes together with its first derivatives on the curves $\gamma_{a}$ and $\gamma_{d}$ (since $\tilde{\varphi_{0}}$ takes value 0 at the ends of $\gamma_{a}$ and $\gamma_{d}$ it is enough to demand that the derivatives vanish). Now we would like to investigate consequences of this condition. Then we have to prove that $\varphi_{0}$ is positive between the curves $\gamma_{a}$ and $\gamma_{d}$.

We first investigate continuity of the derivatives of $\varphi_{0}$ on the left boundary of the oscillon (the curve $\gamma_{a}$ ). After introducing the variables $y_{+}=x+t, y_{-}=x-t$ formula (2.10) takes the form

$$
\varphi_{0}\left(y_{+}, y_{-}\right)=H\left(y_{+}\right)-H\left(y_{-}+2 T\right)+\frac{\lambda}{2} T^{2}-\frac{\lambda}{8}\left(y_{+}-y_{-}\right)^{2}
$$

Setting the derivative of (2.14) with respect to $y_{-}$to zero, we obtain the equation

$$
\chi\left(y_{-}+2 T\right)=\frac{\lambda}{2}\left(y_{+}-y_{-}\right) .
$$

Setting to zero the derivative with respect to $y_{+}$, we have

$$
\chi\left(y_{+}\right)=\frac{\lambda}{2}\left(y_{+}-y_{-}\right),
$$

For solutions we are looking for, (2.15) and (2.16) should describe the same curve $\gamma_{a}$ being the trajectory of the left boundary of the oscillon. This curve joins point $(0,0)$ with the point $(\Delta, T)$. We 
denote by $\alpha$ and $\beta$ the functions

$$
\begin{gathered}
\alpha\left(y_{-}\right)=y_{-}+\frac{2}{\lambda} \chi\left(y_{-}+2 T\right), \Delta-T \leq y_{-} \leq 0, \\
\beta\left(y_{+}\right)=y_{+}-\frac{2}{\lambda} \chi\left(y_{+}\right), \quad 0 \leq y_{+} \leq T+\Delta .
\end{gathered}
$$

Our assumption about the solutions of (2.15) and (2.16) implies that the functions $\alpha$ and $\beta$ are inverse of each other. This should be considered as a condition that the function $\chi$ has to fulfil. It allows to determine the function $\chi$ on one of the intervals $[0, T+\Delta]$ or $[T+\Delta, 2 T]$, provided that it is given on the other. Since $\alpha(-T+\Delta)=T+\Delta$ we have

$$
\chi(T+\Delta)=\lambda T .
$$

Demanding that the trajectory of the oscillon boundary $\gamma_{a}$ is a timelike curve, we obtain the inequalities

$$
\begin{gathered}
\frac{d \chi}{d x}(x)>\frac{\lambda}{2}, \quad 0<x<T+\Delta, \\
\frac{d \chi}{d x}(x)<-\frac{\lambda}{2}, \quad T+\Delta<x<2 T .
\end{gathered}
$$

The equations for the trajectory of the right boundary of the oscillon $\gamma_{d}$ may be obtained after setting derivatives of (2.11) to zero

$$
\begin{gathered}
\chi\left(y_{-}\right)=\frac{\lambda}{2}\left(y_{+}-y_{-}\right), \\
\chi\left(y_{+}-2 T\right)=\frac{\lambda}{2}\left(y_{+}-y_{-}\right) .
\end{gathered}
$$

It is not difficult to see that if $\left(y_{+}, y_{-}\right)$solves $(2.15),(2.16)$, then $\left(y_{+}+2 T, y_{-}+2 T\right)$ solves $(2.22)$, (2.23).

It remains to show that $\varphi_{0}(x, t)$ is positive between the curves $\gamma_{a}$ and $\gamma_{d}$ We begin with the part " $a$ ". The field $\varphi_{0}$ is equal to zero on the curve $\gamma_{a}$. It is enough to show that the derivative

$$
\frac{\partial \varphi_{0}}{\partial x}\left(y_{+}, y_{-}\right)=\frac{1}{2} \chi\left(y_{+}\right)-\frac{1}{2} \chi\left(y_{-}+2 T\right)
$$

is positive inside the part " $a$ ". We denote

$$
\tilde{y}=\alpha\left(y_{-}\right) \equiv y_{-}+\frac{2}{\lambda} \chi\left(y_{-}+2 T\right) .
$$

Since inside the oscillon

$$
y_{+}>\alpha\left(y_{-}\right),
$$

we have $\tilde{y}<y_{+}$and (2.24) may be evaluated in the following way

$$
\begin{aligned}
\frac{\partial \varphi_{0}}{\partial x}\left(y_{+}, y_{-}\right) & =\frac{1}{2} \chi\left(y_{+}\right)-\frac{\lambda}{4}\left(\tilde{y}-y_{-}\right) \\
& =\frac{1}{2} \chi\left(y_{+}\right)-\frac{\lambda}{4}(\tilde{y}-\beta(\tilde{y})) \\
& =\frac{1}{2}\left(\chi\left(y_{+}\right)-\chi(\tilde{y})\right)>0 .
\end{aligned}
$$


Similar reasoning can show that

$$
\frac{\partial \varphi_{0}}{\partial x}\left(y_{+}, y_{-}\right)<0
$$

in the part " $d "$.

From $\chi(0)=\chi(2 T)=0, \chi(T+\Delta)=\lambda T,(2.20)$, and (2.21) it follows that

$$
\begin{gathered}
\chi(x)>\frac{\lambda}{2} x, 0<x \leq T+\Delta, \\
\chi(x)<\lambda T-\frac{\lambda}{2}(T+\Delta-x), \quad 0 \leq x<T+\Delta, \\
\chi(x)>\frac{\lambda}{2}(2 T-x), \quad T+\Delta \leq x<2 T, \\
\chi(x)<\lambda T-\frac{\lambda}{2}(x-T-\Delta), \quad T+\Delta<x \leq 2 T .
\end{gathered}
$$

Using (2.27) one can establish that $\varphi_{0}$ is positive on the set $0 \leq y_{-}<y_{+} \leq T+\Delta$ (triangle " $b$ " in Fig. 1). If $t=\left(y_{+}-y_{-}\right) / 2=0$ the field $\varphi_{0}$ is equal to zero. For $t>0$ we have

$$
\frac{\partial \varphi_{0}}{\partial y_{+}}=\frac{1}{2} \chi\left(y_{+}\right)-\frac{\lambda}{4}\left(y_{+}-y_{-}\right)>\frac{\lambda}{4} y_{-} \geq 0
$$

so $\varphi_{0}(x, t)>0$ for $t>0$. The triangles " $c$ ", " $e$ ", and " $f$ " may be treated in a similar manner.

The last part is the rectangle " $g$ ". Using (2.20), (2.21), and (2.8) it is easy to show that the inequality

$$
\left(\tilde{y}_{+}-y_{+}\right)\left(\frac{\partial \varphi_{0}}{\partial y_{+}}(\tilde{y})-\frac{\partial \varphi_{0}}{\partial y_{+}}(y)\right)+\left(\tilde{y}_{-}-y_{-}\right)\left(\frac{\partial \varphi_{0}}{\partial y_{-}}(\tilde{y})-\frac{\partial \varphi_{0}}{\partial y_{-}}(y)\right)<0
$$

holds for any two points $y$ and $\tilde{y}$ in " $g$ ". It follows that $\varphi_{0}$ is strictly concave in the part " $g$ ". Since it is non-negative on the boundary of the rectangle it is positive inside it.

Let us compute the energy of the oscillon. Since for $t=0$ the field $\varphi$ is equal to zero, the energy is given by the integral

$$
E=\frac{1}{2} \int_{0}^{2 T} \chi(x)^{2} d x
$$

From (2.15)-(2.18) we have

$$
\chi(x)=\left\{\begin{array}{cl}
\frac{\lambda}{2}(x-\beta(x)) & \text { if } 0<x<T+\Delta, \\
\frac{\lambda}{2}(\alpha(x-2 T)-x+2 T) & \text { if } T+\Delta<x<2 T,
\end{array}\right.
$$


Using this and remembering that $\alpha$ and $\beta$ are inverse of each other, one may obtain

$$
\begin{aligned}
E & =\frac{\lambda^{2}}{8} \int_{0}^{T+\Delta}(x-\beta(x))^{2} d x+\frac{\lambda^{2}}{8} \int_{T+\Delta}^{2 T}(\alpha(x-2 T)+x-2 T)^{2} d x \\
& =\frac{\lambda^{2}}{8} \int_{0}^{T+\Delta}(x-\beta(x))^{2} d x-\frac{\lambda^{2}}{8} \int_{0}^{T+\Delta}(x-\beta(x))^{2} \frac{d \beta}{d x} d x \\
& =\frac{\lambda^{2}}{8} \int_{0}^{\frac{2}{\lambda} \chi(T+\Delta)} u^{2} d u=\frac{1}{3 \lambda} \chi(T+\Delta)^{3}=\frac{1}{3} \lambda^{2} T^{3} .
\end{aligned}
$$

We see that the energy depends only on the oscillon size and is the same for the oscillons with different trajectories of the boundaries.

\section{Ending remarks}

In the previous section we have considered a family of periodic solutions of the signum-Gordon equation. It is not difficult to find examples of these solutions. One may use (2.34) to obtain initial data (2.5), (2.6) for an oscillon solution. This requires two bijective functions

$$
\begin{aligned}
& \alpha:[\Delta-T, 0] \rightarrow[0, T+\Delta], \\
& \beta:[0, T+\Delta] \rightarrow[\Delta-T, 0],
\end{aligned}
$$

which are inverse of each other, strictly decreasing, continuous and have a piecewise continuous derivatives.

If these functions are linear

$$
\begin{aligned}
& \alpha(y)=-\frac{T+\Delta}{T-\Delta} y, \\
& \beta(y)=-\frac{T-\Delta}{T+\Delta} y,
\end{aligned}
$$

we get the initial data for oscillons found in [4]. The function $\chi$ is given by the formula

$$
\chi(x)=\left\{\begin{array}{cl}
\frac{\lambda}{1+v} x & \text { if } 0 \leq x \leq T+\Delta \\
\frac{\lambda}{1-v}(x-2 T) & \text { if } T+\Delta \leq x \leq 2 T
\end{array}\right.
$$

where $v=\Delta / T$. The solution for these initial data may be obtained from (2.4), (2.8), (2.10), and (2.11).

To find more examples of the oscillons, we may take any strictly decreasing, sufficiently regular bijection $\alpha$, find the inverse function $\beta$, and then proceed as previously to obtain the periodic solution (the choice of the function $\alpha$ is more limited if we want to evaluate the integral $H(x)=\int \chi(x) / 2 d x$ ). 


\section{Z. Swierczyński / On the oscillons in the signum-Gordon model}

For example, we may choose a homographic function

$$
\alpha(y)=\frac{A(T+\Delta) y}{y-(A-1)(T-\Delta)},
$$

where $A$ is a constant, $A>1$ or $A<0$. The inverse function is also a homographic function

$$
\beta(y)=\frac{(A-1)(T-\Delta) y}{y-A(T+\Delta)} .
$$

The function $H$ is given by the formulae

$$
\begin{aligned}
H(x)= & \frac{\lambda}{8}\left(x^{2}-(T+\Delta)^{2}\right)-\frac{\lambda}{4}(A-1)(T-\Delta)(x-T-\Delta) \\
& -\frac{\lambda}{4} A(A-1)\left(T^{2}-\Delta^{2}\right) \ln \left|\frac{x-A(T+\Delta)}{(A-1)(T+\Delta)}\right|
\end{aligned}
$$

if $0 \leq x \leq T+\Delta$,

$$
\begin{aligned}
H(x)= & \frac{\lambda}{8}\left((T-\Delta)^{2}-(x-2 T)^{2}\right)+\frac{\lambda}{4} A(T+\Delta)(x-T-\Delta) \\
& +\frac{\lambda}{4} A(A-1)\left(T^{2}-\Delta^{2}\right) \ln \left|\frac{x-T-\Delta-A(T-\Delta)}{A(T-\Delta)}\right|
\end{aligned}
$$

if $T+\Delta \leq x \leq 2 T$. Figure 3 shows the shapes of the oscillon obtained for $\lambda=1, T=1, \Delta=-1 / 2$, $A=11 / 10$. The function $\alpha$ is given by the formula

$$
\alpha(y)=\frac{11 y}{20 y-3} .
$$

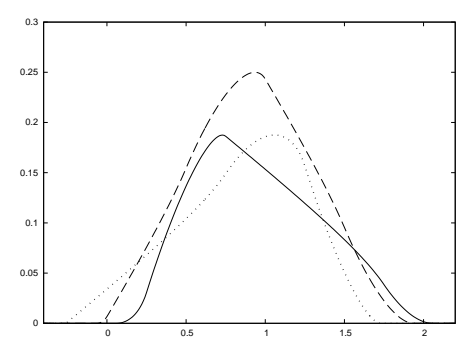

Fig. 3. The shape of the oscillon obtained at the times $t=1 / 8$ (solid line), $t=1 / 2$ (dashed line), and $t=7 / 8$ (doted line); $\alpha(y)=11 y /(20 y-3)$.

Another simple example of our new oscillon solutions is obtained if we choose a piecewise linear function $\alpha$. Then the functions $\beta$ and $\chi$ are also piecewise linear functions. Integrating the function $\chi$ we obtain a $C^{1}$ class, piecewise quadratic function $H$.

\section{Acknowledgements}

We would like to thank H. Arodź for useful remarks on the manuscript of this paper. We also thank the Referees for very valuable remarks. 


\section{References}

[1] H. Arodź, P. Klimas and T. Tyranowski, Scaling, self-similar solutions and shock waves for V-shaped field potentials, Phys. Rev. E 73 (2006) 046609.

[2] H. Arodź and J. Lis, Compact Q-balls and Q-shells in a scalar electrodynamics, Phys. Rev. D 79 (2009) 045002.

[3] H. Arodź, P. Klimas and T. Tyranowski, Compact oscillons in the signum-Gordon model, Phys.Rev. D 77 (2008) 047701.

[4] H. Arodź and Z. Świerczyński, Swaying oscillons in the signum-Gordon model, Phys. Rev. D 84 (2011) 067701.

[5] I. L. Bogolyubskii and V. G. Makhankov, Lifetime of pulsating solitons in certain classical models, JETP Lett. 24 (1976) 12-14.

[6] J. P. Boyd, Weakly Nonlocal Solitary Waves and Beyond-All-Orders Asymptotics, Mathematics and Its Applications (Springer Science+Business Media, Dordrecht, 1998).

[7] J. Denzler, A hypergeometric function approach to the persistence problem of single sine-Gordon breathers, Trans. Amer. Math. Soc. 349 (1997) 4053-4083.

[8] G. Fodor, P. Forgács, P. Grandclément and I. Rácz, Oscillons and quasibreathers in the $\Phi^{4}$ Klein-Gordon model, Phys. Rev. D 74 (2006) 124003.

[9] G. Fodor, P. Forgács, Z. Horváth, A. Lukács, Small amplitude quasibreathers and oscillons, Phys.Rev. $D 78$ (2008) 025003.

[10] G. Fodor, P. Forgács, Z. Horváth, M. Mezei, Computation of the radiation amplitude of oscillons, Phys. Rev. D 79, (2009) 065002.

[11] G. Fodor, P. Forgács, Z. Horváth, M. Mezei, Radiation of scalar oscillons in 2 and 3 dimensions, Physics Letters B 674, (2009) 319-324.

[12] M. Gleiser, d-Dimensional oscillating scalar field lumps and the dimensionality of space, Phys. Lett. $B$ 600 (2004) 126-132.

[13] M. Gleiser and D. Sicilia, Analytical characterization of oscillon energy and lifetime, Phys. Rev. Lett. 101 (2008) 011602.

[14] M. Gleiser and J. Thorarinson, Phase transition in U(1) configuration space: Oscillons as remnants of vortex-antivortex annihilation, Phys. Rev. D 76 (2007) 041701(R).

[15] M. Hindmarsh and P. Salmi, Numerical investigations of oscillons in 2 dimensions, Phys. Rev. D 74 (2006) 105005.

[16] S. Kichenassamy, Breather solutions of the nonlinear wave equation, Comm. Pur. Appl. Math., 44, 789818 (1991).

[17] M. Kruskal and H. Segur, Nonexistence of small-amplitude breather solutions in $\varphi^{4}$ theory, Phys. Rev. Lett. 58 (1987) 747-750. 